Review began 01/14/2022 Review ended 01/29/2022 Published 02/03/2022

\section{() Copyright 2022}

Khan et al. This is an open access article distributed under the terms of the Creative Commons Attribution License CC-BY 4.0. which permits unrestricted use, distribution, and reproduction in any medium, provided the original author and source are credited.

\title{
Interstitial Lung Disease and Transverse Myelitis: A Possible Complication of COVID-19 Vaccine
}

\author{
Zunaira Khan ${ }^{1}$, Ahmad Ammar Khattak ${ }^{2}$, Nawal Rafiq ${ }^{3}$, Anam Amin ${ }^{4}$, Mahwish Abdullah ${ }^{5}$ \\ 1. Accident and Emergency, Kingston Hospital NHS Foundation Trust, London, GBR 2. Pathology, Kabir Medical \\ College, Gandhara University, Peshawar, PAK 3. Accident and Emergency, Rehman Medical Institute, Peshawar, PAK 4. \\ Internal Medicine, Northwest General Hospital, Peshawar, PAK 5. Anesthesia, Pakistan Air Force Hospital, Islamabad, \\ PAK \\ Corresponding author: Nawal Rafiq, nawarafiq10@gmail.com
}

\begin{abstract}
The clinical impact of the severe acute respiratory syndrome 2 (SARS-CoV-2) pandemic is growing, and vaccine-associated complications are becoming more evident. Although global vaccination against coronavirus disease 19 (COVID-19) is an outstanding accomplishment, safety concerns and adverse outcomes are also emerging that need to be addressed promptly. The most reported side effects of the COVID-19 vaccine include fever, myalgia, headache, and injection site reactions. Acute transverse myelitis (ATM) and interstitial lung disease (ILD) following the CoronaVac vaccine are rarely reported. We report a case of ILD followed by acute myelopathy in a female who presented with dyspnea, cough, and fever after the second dose of the COVID-19 vaccine. On the third day of admission, she developed paresthesia and bilateral upper and lower limb weakness. She was diagnosed with ILD and ATM due to the COVID-19 vaccine based on imaging and detailed investigations after ruling out all possible causes. Her neurological and respiratory manifestations improved gradually after starting intravenous methylprednisolone.
\end{abstract}

Categories: Internal Medicine, Infectious Disease, Pulmonology

Keywords: vaccine adverse events, covid 19 vaccine, acute transverse myelitis (atm), acute myelopathy, interstitial lung disease

\section{Introduction}

Acute transverse myelitis (ATM) is an immune-mediated condition characterized by spinal cord inflammation, manifesting as autonomic, motor, or sensory deficits below the lesion level [1]. ATM can affect any spinal cord segment, with the cervical region being the most affected segment, followed by the thoracic region. ATM can be divided into non-compressive and compressive myelopathies, and noncompressive causes include idiopathic, infectious, post-vaccination, post-infectious, and paraneoplastic syndromes [2]. The most common etiology of ATM is idiopathic, and the infections causing ATM include human immunodeficiency virus, cytomegalovirus, herpes viruses, neuroborreliosis, mycoplasma, Zika Virus, and West Nile virus [3]. A meta-analysis reported 37 cases presenting signs and symptoms of ATM due to different vaccines, including hepatitis B, diphtheria, measles-mumps-rubella, polio, and other vaccines [4].

Interstitial lung disease (ILD) has a broad spectrum of clinical presentations ranging from transient lung inflammation to acute respiratory distress syndrome and manifests with ground-glass opacities with or without consolidation in peripheral, basal, or bilateral areas in multiple lobes [5]. ILD and ATM after vaccination are also underlined in the literature [6]. Even though ILD and ATM are sporadic disorders commonly associated with severe acute respiratory distress syndrome 2 (SARS-CoV-2), ILD and ATM due to coronavirus disease 19 (COVID-19) vaccination are rare, and a few cases have been reported [7,8]. We report a case of ILD and ATM caused by the COVID-19 vaccination in a single patient.

\section{Case Presentation}

A 61-year-old female with a past medical history of asthma and hypertension was admitted to the emergency department with complaints of dyspnea, cough, and fever for the last two days. She reported that her symptoms began after receiving the second dose of the CoronaVac vaccine two days ago. She was on the albuterol-beclomethasone inhaler for asthma and amlodipine for hypertension, and she reported compliance with her medications. She did not report any change in medications in the last six months. Initially, she had fever, nausea, and myalgia and took acetaminophen for symptomatic improvement. She had no allergies and denied smoking, alcohol abuse, and illicit drug use.

On initial evaluation, her vitals were stable except for tachypnea and low oxygen saturation. Chest X-ray revealed infiltrates in both lungs, and computed tomography (CT) of the chest demonstrated multiple asymmetrical ground-glass opacities in both lungs (Figure 1). The results of the initial laboratory analysis were normal except for elevated c-reactive protein $(17.20 \mathrm{mg} / \mathrm{dL})$, lactate dehydrogenase $(302 \mathrm{U} / \mathrm{L})$, and ddimer $(1.1 \mathrm{mg} / \mathrm{L})$. Sputum cytology did not reveal any organism. Legionella pneumophilia antigen and pneumococcal antigen testing were negative. A quantitative test for COVID-19 antigen on admission was 


\section{Cureus}

also negative. She was initially managed with supplemental oxygen. Her condition worsened gradually, for which she required mechanical ventilation. She was commenced on azithromycin and ampicillin/sulbactam based on the provisional diagnosis of pneumonia.

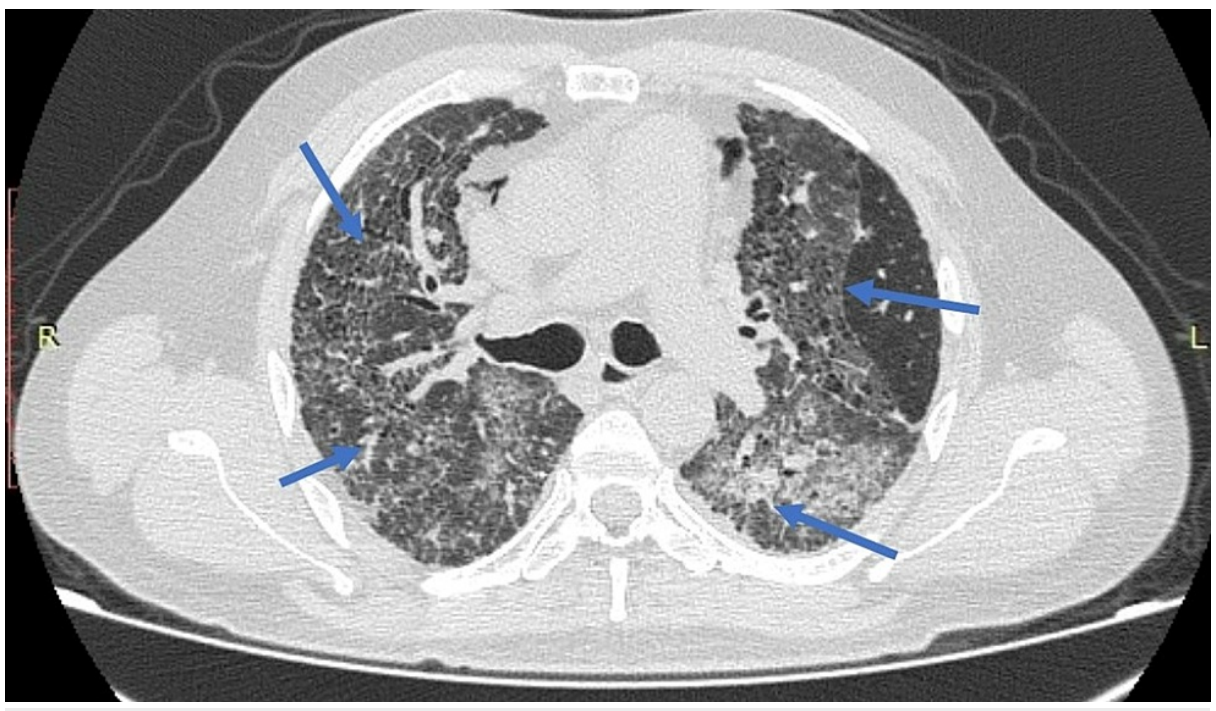

FIGURE 1: CT chest showing interstitial infiltrates in both lung fields.

CT: Computed tomography

She reported improvements in her respiratory system, and she was extubated on the second day of admission. She reported sudden onset of abnormal sensations and weakness in bilateral upper and lower limbs on the third day. Neurological examination revealed upper motor neuron disease signs, including hyperreflexia, hypertonia, and spasticity, in both upper and lower extremities with increased severity in legs. She also had hypoesthesia in the upper and lower limbs. Cranial nerves were intact, and there were no signs of meningeal irritation. She denied diplopia, facial weakness, neck stiffness, dysarthria, or dyspnea. Magnetic resonance imaging (MRI) of the whole spine was performed, which showed an expansile central hyperintense signal in the cervical segment of the spinal cord (Figure 2). Brain MRI showed age-related changes. Cerebrospinal fluid (CSF) analysis revealed no abnormality (Table 1).

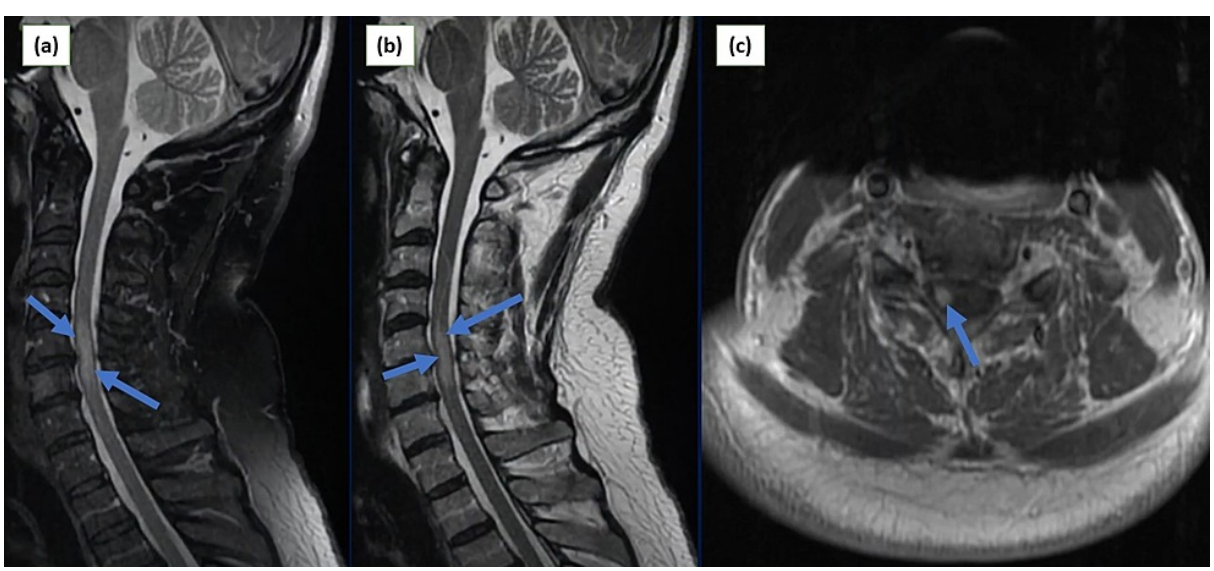

FIGURE 2: Cervical MRI images: (a) sagittal view demonstrating T1weighted image with signal enhancement from C4-C6; (b) sagittal view showing T2-weighted image with hyperintense signals from C3-C5; (c) axial view of cervical spine demonstrating uniform enhancement at the level of $\mathbf{C 4 .}$

MRI: magnetic resonance imaging, C: cervical. 


\section{Cureus}

\begin{tabular}{|c|c|c|}
\hline CSF analysis & Lab result & Reference \\
\hline RBC & Not detected & $<1 / \mathrm{mm}^{3}$ \\
\hline Nucleated cell & 2 & $<8 / \mathrm{mm}^{3}$ \\
\hline Protein & 40 & $<45 \mathrm{mg} / \mathrm{dl}$ \\
\hline Glucose & 67 & $40-70 \mathrm{mg} / \mathrm{dl}$ \\
\hline Color & Colorless & Colorless \\
\hline Appearance & Clear & Clear \\
\hline LDH & 21 & $<40 \mathrm{U} / \mathrm{L}$ \\
\hline
\end{tabular}

TABLE 1: The result of CSF analysis.

RBC: red blood cell, LDH: lactate dehydrogenase, CSF: cerebrospinal fluid.

The results of autoimmune screening, including antinuclear antibodies, rheumatoid factor, anticardiolipin, and atypical antibodies, including aquaporin-4 antibodies (AQP4-ab), were negative. Infectious workup for human immunodeficiency virus, herpesvirus, varicella-zoster virus, and syphilis serology was also negative. The findings were suggestive of transverse myelitis.

She was started on intravenous methylprednisolone $1 \mathrm{~g} / \mathrm{kg} /$ day along with the current management, and physical therapy was initiated. She reported improvement in sensation in the affected limbs. She was discharged to a rehabilitation facility with aggressive physical and occupational therapy. At her recent follow-up two weeks later, she had started walking with a cane, and a significant improvement in her condition was noted. She is currently on low-dose methylprednisolone and improving well with no further exacerbations.

\section{Discussion}

Despite the excellence of this achievement amid the pandemic, post-marketing surveillance and adverse outcomes associated with the COVID-19 vaccines are still required [6,9]. Reports of COVID-19 vaccineassociated adverse effects emerged after the vaccines became widely available [10]. Significant complications related to the COVID-19 vaccine include seizures, stroke, acute disseminated encephalomyelitis, pericarditis, pancreatitis, acute respiratory distress syndrome [11]. The most-reported neurological side effects of the COVID-19 vaccine include headache, dizziness, myalgia, and paresthesia [12]. A small number of ATM cases caused by COVID-19 vaccination have also been underlined [13,14]. Tahir et al. reported a case of ATM after the COVID-19 vaccine, and the latency period between vaccination and onset of symptoms was 10 days [13]. Similarly, Khan et al. also highlighted an ATM case after Moderna COVID-19 vaccination [14]. Likewise, cases of ILD after COVID-19 vaccines have also been reported [15]. Yoshifuji et al. described a case of ILD after vaccination [15]. Kono et al. also reported a case of ILD after two weeks of COVID-19 immunization [16].

COVID-19 and influenza virus vaccines are commonly linked to ILD and ATM, and many sporadic cases of ILD and ATM due to vaccinations have also been reported [10,17]. Pathophysiology of vaccine-induced complications is molecular mimicry, and vaccine adjuvants can induce autoimmunity similar to infectious agents [5]. An acute autoimmune reaction occurs in molecular mimicry due to cross-reactivity with structurally related host proteins [6]. Hyperinflammatory response, cytokine upregulation, epitope spreading, and activation of B and T cells due to the COVID-19 vaccine can lead to the occurrence of neurological and respiratory manifestations by stimulating immune reactions [18]. A recently published article underlined the immunological reactions between COVID-19 spike protein antibodies and host proteins and their interactions with myelin basic protein that might contribute to the pathogenesis of the demyelinating autoimmune disease [19,20]. Furthermore, angiotensin-converting enzyme 2 (ACE2) receptors are expressed in the respiratory tract, gastrointestinal, and nervous systems, particularly in the blood-brain barrier. Their interaction with the viral spike proteins can also trigger the hyperinflammatory response [20].

In our case, the patient was diagnosed with ILD and ATM. Though she had bronchial asthma and hypertension, she had been stable for several years and had no drug reactions or underlying pulmonary and neurological disease. Therefore, we consider the COVID-19 vaccine could trigger ILD and ATM. Additionally, her symptoms improved gradually after commencing methylprednisolone. We assume that the chronological association of vaccines with the development of ATM, and ILD, the absence of any infectious etiology, and 
the effective clinical response to steroids support the notion that the COVID-19 vaccine is the etiology of both ILD and ATM.

\section{Conclusions}

To our knowledge, we present the first case of ILD and ATM due to the COVID-19 vaccine in a single patient, and our case highlights the association of the COVID-19 vaccine with the development of ILD and ATM. Diagnosis requires a high index of suspicion in patients manifesting atypical symptoms after COVID-19 vaccination. Our case also warrants further study to establish the causality and association between the COVID-19 vaccine and the occurrence of vaccine complications, including ATM and ILD.

\section{Additional Information \\ Disclosures}

Human subjects: Consent was obtained or waived by all participants in this study. Conflicts of interest: In compliance with the ICMJE uniform disclosure form, all authors declare the following: Payment/services info: All authors have declared that no financial support was received from any organization for the submitted work. Financial relationships: All authors have declared that they have no financial relationships at present or within the previous three years with any organizations that might have an interest in the submitted work. Other relationships: All authors have declared that there are no other relationships or activities that could appear to have influenced the submitted work.

\section{Acknowledgements}

The authors would like to thank the patient for her consent and providing details for the case report.

\section{References}

1. Beh SC, Greenberg BM, Frohman T, Frohman EM: Transverse myelitis. Neurol Clin. 2013, 31:79-138. 10.1016/j.ncl.2012.09.008

2. Krishnan C, Kaplin AI, Deshpande DM, Pardo CA, Kerr DA: Transverse myelitis: pathogenesis, diagnosis and treatment. Front Biosci. 2004, 9:1483-1499. 10.2741/1351

3. Dumic I, Vitorovic D, Spritzer S, Sviggum E, Patel J, Ramanan P: Acute transverse myelitis - A rare clinical manifestation of Lyme neuroborreliosis. IDCases. 2019, 15:e00479. 10.1016/j.idcr.2018.e00479

4. Zanoni G, Nguyen TM, Destefani E, Masala L, Nardelli E, Tridente G: Transverse myelitis after vaccination. Eur J Neurol. 2002, 9:696-697. 10.1046/j.1468-1331.2002.00447 9.x

5. Skeoch S, Weatherley N, Swift AJ, et al.: Drug-induced interstitial lung disease: a systematic review . J Clin Med. 2018, 7:356. 10.3390/jcm7100356

6. Voysey M, Clemens SA, Madhi SA, et al.: Safety and efficacy of the ChAdOx1 nCoV-19 vaccine (AZD1222) against SARS-CoV-2: an interim analysis of four randomised controlled trials in Brazil, South Africa, and the UK. Lancet. 2021, 397:99-111. 10.1016/S0140-6736(20)32661-1

7. Park JY, Kim JH, Lee IJ, et al.: COVID-19 vaccine-related interstitial lung disease: a case study . Thorax. 2022, 77:102-104. 10.1136/thoraxjnl-2021-217609

8. Gao JJ, Tseng HP, Lin CL, Shiu JS, Lee MH, Liu CH: Acute transverse myelitis following COVID-19 vaccination. Vaccines (Basel). 2021, 9:1008. 10.3390/vaccines9091008

9. Knoll MD, Wonodi C: Oxford-AstraZeneca COVID-19 vaccine efficacy . Lancet. 2021, 397:72-74. 10.1016/S0140-6736(20)32623-4

10. Kaur RJ, Dutta S, Bhardwaj P, et al.: Adverse events reported from COVID-19 vaccine trials: a systematic review. Indian J Clin Biochem. 2021, 36:427-439. 10.1007/s12291-021-00968-z

11. Menni C, Klaser K, May A, et al.: Vaccine side-effects and SARS-CoV-2 infection after vaccination in users of the COVID Symptom Study app in the UK: a prospective observational study. Lancet Infect Dis. 2021, 21:939-949. 10.1016/S1473-3099(21)00224-3

12. Goss AL, Samudralwar RD, Das RR, Nath A: ANA investigates: neurological complications of COVID-19 vaccines. Ann Neurol. 2021, 89:856-857. 10.1002/ana.26065

13. Tahir N, Koorapati G, Prasad S, Jeelani HM, Sherchan R, Shrestha J, Shayuk M: SARS-CoV-2 vaccinationinduced transverse myelitis. Cureus. 2021, 13:e16624. 10.7759/cureus.16624

14. Khan E, Shrestha AK, Colantonio MA, Liberio RN, Sriwastava S: Acute transverse myelitis following SARSCoV-2 vaccination: a case report and review of literature (PREPRINT). J Neurol. 2021, 10.1007/s00415-02110785-2

15. Yoshifuji A, Ishioka K, Masuzawa Y, et al.: COVID-19 vaccine induced interstitial lung disease . J Infect Chemother. 2022, 28:95-98. 10.1016/j.jiac.2021.09.010

16. Kono A, Yoshioka R, Hawk P, et al.: A case of severe interstitial lung disease after COVID-19 vaccination . QJM. 2021, 114:805-806. 10.1093/qjmed/hcab263

17. Umeda Y, Morikawa M, Anzai M, Sumida Y, Kadowaki M, Ameshima S, Ishizaki T: Acute exacerbation of idiopathic pulmonary fibrosis after pandemic influenza A (H1N1) vaccination. Intern Med. 2010, 49:23332336. 10.2169/internalmedicine.49.3890

18. Matsuno O: Drug-induced interstitial lung disease: mechanisms and best diagnostic approaches . Respir Res. 2012, 13:39. 10.1186/1465-9921-13-39

19. Bottazzi ME, Strych U, Hotez PJ, Corry DB: Coronavirus vaccine-associated lung immunopathology-what is the significance?. Microbes Infect. 2020, 22:403-404. 10.1016/j.micinf.2020.06.007

20. McDonald I, Murray SM, Reynolds CJ, Altmann DM, Boyton RJ: Comparative systematic review and metaanalysis of reactogenicity, immunogenicity and efficacy of vaccines against SARS-CoV-2. NPJ Vaccines. 


\section{Cureus}

2021, 6:74. 10.1038/s41541-021-00336-1 